\title{
LEGISLACIÓN CHILENA DE PENSIONES E INDICACIONES DE LA COMISIÓN DE EXPERTOS DE LA OIT
}

\author{
DR. PABlo ARELlano ORTIZ* \\ Departamento de Normas OIT \\ DR. Hugo Cifuentes Lillo \\ Pontificia Universidad Católica de Chile
}

\begin{abstract}
RESUMEN: El artículo analiza las últimas modificaciones al sistema de pensiones, contenidas en la Ley No 20.255, a la luz de las exigencias de la Comisión de Expertos de la Organización Internacional del Trabajo, de acuerdo con la recomendación No 35, ratificada por Chile en 1935. En particular, si el sistema de pensiones chileno está administrado por organizaciones que no persiguen fines de lucro; participación de los asegurados en la gestión del sistema, y la contribución de los empleadores a su financiamiento.
\end{abstract}

Palabras clave: Sistema de pensiones, Convenio No 35 OIT, recomendaciones, Comisión de Expertos.

ABSTRACT: The article analyzes the last modifications to the system of pensions, wich are contained in the law number 20. 255, from the perspective of the demands of the "Comisión de Expertos de la Organización Internacional del Trabajo", acording with the recomendation number 35, ratified by Chile in 1935. Particularly, if the chilean system of pensions is administered by non-profit organizations; the participation of those assured in the management of the system and the contribution of the employers in the financing.

Key words: System of pensions, Agreement number 35 OIT, recomendations, Experts Comission.

\section{PRELIMINARES}

El sistema de protección de la vejez, invalidez y sobrevivencia chileno, establecido mediante el Decreto Ley 3.500 de 1980, ha sido objeto de observaciones por no cumplir con las normas establecidas en el Convenio $\mathrm{N}^{\circ} 35$ de la OIT, ratificado por Chile el 18 de octubre de 1935. La Comisión de Expertos sobre Aplicación de Convenios y Recomendaciones $^{1}$ ha insistido recientemente en realizar observaciones acotando este problema de incumplimiento con el Convenio indicado a tres puntos específicos a fin de determinar si la legislación chilena se encuentra conforme o no a dicho instrumento internacional. Sin embargo, estas recomendaciones en realidad fueron adoptadas por el Consejo de Administración en su $277^{a}$ reunión en marzo del año $2000^{2}$.

\footnotetext{
* Las opiniones emiridas por el autor en el presente artículo se expresan estrictamente a título personal. No representan ni son vinculantes para con la organización en donde actualmente se desempeña.

${ }^{1}$ Ver Comisión de Expertos sobre Aplicación de Convenios y Recomendaciones (CEACR): Observación individual sobre el Convenio sobre el seguro de vejez (industria, etc.), 1933 (No 35) Chile (ratificación: 1935) Publicación: 2009, documento Ilolex número 062009 CHL035.

2 Documento GB 277/17/5. Este documento, así como todos aquellos relativos a las reuniones del Consejo de Administración, se pueden encontrar en el sitio http://www.ilo.org, en la sección relativa a dicho Consejo, ordenados por año y por sesión.
} 
Las recomendaciones establecidas en año 2000 y reiteradas en el 2009 son: i) que el sistema de pensiones establecido por el DL 3.500, en 1980 , sea administrado por instituciones que no persigan fines de lucro; ii) que los representantes de los asegurados participen en la gestión del sistema en las condiciones que determine la legislación y las prácticas locales; y iii) que los empleadores contribuyan al financiamiento. Recientemente (2008), Chile ha reformado el sistema de protección de vejez, invalidez y sobrevivencia, incorporando, entre otros cambios, un mecanismo no contributivo. Por tanto, procederemos a comentar las exigencias de la Comisión de Expertos a la luz de la reciente reforma, incluyendo referencias necesarias a algunas modificaciones experimentadas por el sistema en las dos últimas décadas.

Antes de estudiar cada punto por separado debemos señalar que la reforma de $2008^{3}$ incorporó importantes cambios tanto a nivel del régimen de capitalización individual, como especialmente, en cuanto a establecer un régimen de pensiones solidarias, equivalente a lo que en doctrina se conoce como pilar solidario ${ }^{4}$. Uno de los principales objetivos de la reforma es resolver aspectos de cobertura y de los niveles de las prestaciones, conservando al mismo tiempo las cuentas de capitalización individual gestionadas por las Administradoras de Fondos de Pensiones. La reforma de 2008, a través de la Ley 20.255, introdujo un régimen de pensiones solidarias de vejez e invalidez, denominado "Sistema de Pensiones Solidarias", complementario del régimen general, el cual se financia con recursos del Estado, es decir, se trata de prestaciones no contributivas. Este sistema otorga beneficios de pensiones básicas solidarias de vejez e invalidez y aportes previsionales de vejez e invalidez y es administrado por un servicio público, el Instituto de Previsión Social, IPS. Al final del proceso de aplicación gradual iniciado en julio de 2008 y que culmina en 2011 , serán beneficiarios del régimen todas las personas con 65 o más años de edad que pertenezcan a los tres primeros quintiles de ingresos ( $60 \%$ de la población) y que acrediten residencia en el territorio, por un número determinado de años, según el tipo de pensión.

Tradicionalmente, se ha categorizado al sistema chileno de fondos de pensiones como un sistema que encaja dentro de la estructura multipilar ideada por el Banco Mundial.

En un sistema multipilar cada pilar responde a una lógica propia de protección y que además protege a categorías específicas de la población. Hoy en día, con la reforma de 2008 dicha afirmación se ve disminuida. Por un lado los elementos del modelo chileno de pensiones se pueden encasillar fácilmente en un esquema multipilar en donde la pensión solidaria es el pilar cero (que puede llegar a beneficiar al $60 \%$ de la población), la capitalización obligatoria el primer pilar obligatorio, las contribuciones voluntarias tanto individuales como colectivas constituyen un segundo pilar, esta vez voluntario y complementario del pilar obligatorio. Pero desde otro punto de vista es posible sostener que el sistema

\footnotetext{
3 Para un estudio más exhaustivo de la reforma véanse Cifuentes Lillo, Hugo; Walker ERrAZuriz, Francisco, "Aspectos Generales de la Ley No 20.255 que establece una Reforma Previsional", en: Revista Laboral Chilena, N $^{\circ} 165$, Santiago, 2008. pp. 88-127. CifUENTES Lillo, Hugo (coordinador), Reforma previsional, Editorial LexisNexis, Santiago, 2008.

4 Sobre esta materia léase ARELlano ORTIZ, Pablo, "El pilar solidario, la solución esperada para nuestro sistema de AFP", en: Revista Laboral Chilena, $\mathrm{N}^{\circ} 164$, Santiago, 2008, pp. 86-88.

${ }^{5}$ Para una aproximación a esta opinión véase ARELLANO ORTIZ, Pablo, Universalisme et individualisme dans le droit chilien des retraites. Thèse, Université Paris Ouest La Défense, 2009. Él mismo, "El pilar solidario... (n. 4).
} 
chileno ya no sigue el esquema de un modelo multipilar constituyendo un sistema único de protección de la vejez ${ }^{5}$. Si bien existen diferentes mecanismos (no contributivos, contributivo obligatorio y contributivo voluntario), el sistema de pensiones chileno posee una lógica general: la capacidad de ahorro individual. Esta capacidad determina qué mecanismo es aplicable a un caso concreto, así una persona sin capacidad de ahorro recibirá una pensión solidaria; una persona con una débil o una mediana capacidad de ahora recibirá el aporte solidario; y aquellos que poseen una importante capacidad de ahorro podrán realizar aportes voluntarios y gozar de los beneficios de este. La reforma de 2008 viene a reforzar el elemento básico del sistema chileno de pensiones: el esfuerzo individual mediante el ahorro, que es complementado de la forma indicada.

El régimen de pensiones solidarias refundió, armonizó y mejoró los regímenes de pensiones asistenciales y de pensiones mínimas garantizadas, existentes antes de la plena vigencia de la Ley 20.255. Dichos mecanismos adolecían de ser programas con una gran dependencia del presupuesto público, bajo condiciones de existencia de recursos económicos y con un gran déficit de cobertura y no estaban concebidos como derechos, sino como beneficios. El nuevo mecanismo no contributivo establece dos tipos de prestaciones:

\subsection{PENSION BÁSICA SOLIDARIA DE VEJEZ E INVALIDEZ (PBS).}

Para ser beneficiario de esta pensión se debe cumplir con los siguientes requisitos: i) no tener derecho a pensión en ningún régimen previsional; ii) haber cumplido 65 años de edad; iii) integrar un grupo familiar perteneciente al $60 \%$ más pobre de la población; y iv) acreditar residencia en el país por 20 años a lo menos, sean continuos o discontinuos, de los cuales cuatro deben estar incluidos en los últimos cinco años inmediatamente anteriores a la solicitud de pensión. El indicado período de residencia se acredita desde que la persona cumple 20 años de edad, con excepción de quienes califican como carentes de recursos, para quienes el señalado período se cuenta desde su nacimiento. El monto de la pensión a contar del 1 de julio de 2009 asciende a $\$ 75.000$ (US\$150 aproximadamente).

\subsection{APORTE PREVISIONAL SOLIDARIO DE VEJEZ E INVALIDEZ (APS)}

Esta prestación consiste en un aporte mensual que complementa las pensiones otorgadas o generadas por el régimen de capitalización individual (DL No 3.500 de 1980), cuando ellas se encuentran bajo un determinado nivel de ingreso. Para acceder al aporte se requiere cumplir los siguientes requisitos: tener derecho a una o más pensiones regidas por el DL 3.500, cuyo monto sea inferior al valor máximo de la pensión con aporte solidario y reunir las condiciones exigid̦as para acceder a Pensión Básica de Vejez de Invalidez, según el caso. El APS ascenderá a la cantidad que resulte de restar de la pensión final, la pensión o suma de pensiones que perciba el solicitante de conformidad al régimen de capitalización individual, del valor de la Pensión Básica de Vejez o Invalidez. El aporte solidario decrece a mayor pensión hasta extinguirse para aquellas de un monto igual o superior a $\$ 255.000$ (US\$510, aproximadamente).

Efectuada esta breve enunciación de las principales modificaciones de la reforma de 2008 en materia de cobertura, analizaremos a continuación las exigencias de la Comisión de Expertos. 


\section{EXIGENCIAS DE LA COMISIÓN DE EXPERTOS}

\subsection{ADMINISTRACIÓN POR UNA INSTITUCIÓN QUE NO PERSIGA FINES DE LUCRO}

Debemos desde ya señalar que la reforma de 2008 viene a validar la existencia de las Administradoras de Fondos de Pensiones (AFP) como mecanismo de gestión del régimen general por capitalización individual. La lógica del sistema de protección chileno de pensión es la capacidad de ahorro. Quien puede ahorrar (cotizar) está obligado por ley a incorporarse a una AFP, que es una sociedad anónima, según el artículo 23 del DL 3.500 , por lo que el fin de lucro es implícito en estas entidades.

No obstante lo anterior, debemos señalar que de acuerdo con la nueva orgánica del sistema chileno, las AFP ya no son las únicas instituciones que participan en la administración del total de sistema. La Ley 20.255 crea el Instituto de Previsión Social (IPS), el cual junto con asumir parte de las funciones que tenía el extinguido Instituto de Normalización Previsional (INP) (gestión de los antiguos regímenes de previsionales de reparto), es el encargado de administrar el denominado Sistema Solidario, de conceder las pensiones de dicho sistema, suspenderlas y extinguirlas, según corresponda.

El IPS es un servicio público descentralizado con personalidad jurídica y patrimonio propio, bajo la supervigilancia del Presidente de la República, a través del Ministerio del Trabajo y Previsión Social, por intermedio de la Subsecretaría de Previsión Social. La dirección superior y la administración del Instituto corresponde a un Director Nacional, que es el jefe superior del servicio y tiene la autoridad y atribuciones y deberes inherentes a esa calidad. La designación de este alto funcionario es efectuada mediante un proceso de selección de alta Dirección Pública ${ }^{6}$.

Las AFP y el IPS se encuentran bajo la vigilancia y control de una Superintendencia de Pensiones (SUPEN), continuadora legal de la antigua Superintendencia de AFP. Esta SUPEN es un organismo público descentralizado, con personalidad jurídica y patrimonio propio, que se rige por las normas de la Ley 20.255 y su estatuto orgánico contenido en Decreto con Fuerza de Ley No 101 de 1980 de Trabajo y Previsión.

A partir de la reforma de 2008 , la consideración sobre el fin de lucro en la administración solo debe hacerse en referencia a quienes integran el régimen general por capitalización individual y reciban pensiones de una AFP o Compañía de Seguro. En caso contrario, es decir, la gestión de las prestaciones solidarias, la administración es pública.

\subsection{PARTICIPACIÓN DE LOS ASEGURADOS EN LA GESTIÓN DEL SISTEMA}

En lo que concierne a este tema la reforma de 2008 ha incorporado tres comisiones participativas: la Comisión de Usuarios, el Consejo Técnico de Inversiones y el Consejo Consultivo Previsional.

La "Comisión de Usuarios del Sistema de Pensiones"7 constituye una instancia relevante de participación y diálogo social. Se integra por un representante de los trabajadores, de los pensionados (en ambos casos de las organizaciones más representativas), de

\footnotetext{
${ }^{6}$ Ley $\mathrm{N}^{\circ} 19.882$.

${ }^{7}$ Comisión creada por el artículo 43 de la Ley $\mathrm{N}^{\circ} 20.255$.
} 
las instituciones públicas de previsión, de las entidades privadas de pensiones y de un académico universitario, que la preside. Las funciones principales de la Comisión incluyen informar a la Subsecretaría de Previsión Social y a otros organismos públicos del sector, sobre las evaluaciones que sus representados efectúen del funcionamiento del Sistema de Pensiones y propone, también, estrategias de educación y difusión del mismo. La Subsecretaría de Previsión Social otorga la asistencia administrativa para el funcionamiento de la Comisión, la que puede pedir apoyo técnico a los organismos públicos correspondientes. Debemos constatar que esta Comisión no es solo tripartita, sino que además el mundo académico está presente, tanto trabajadores activos como pensionados, lo que sin duda constituye un gran avance en materia de diálogo social. Esta Comisión tiene funciones exclusivamente indicativas hacia la autoridad política y técnica del sector y sin implicancia alguna en la administración del sistema.

Se asigna también a la Comisión de Usuarios un rol propositivo en materia de formación y educación a la población en materia de previsión social y seguridad social en general.

El "Consejo Técnico de Inversiones" también fue creado por la Ley 20.255, tiene por objeto evacuar informes, propuestas y pronunciamientos respecto de las inversiones de los Fondos de Pensiones, con el objeto de procurar una adecuada rentabilidad y seguridad de estos. El Consejo cuenta, entre otras, las siguientes funciones y atribuciones:

1) Pronunciarse sobre el contenido del Régimen de Inversión de los Fondos y sobre las modificaciones que la Superintendencia de Pensiones proponga efectuar al mismo;

2) Emitir opinión técnica en materias de inversiones, en especial respecto de la estructura de límites de los Fondos contenidas en el Régimen de Inversión;

3) Efectuar propuestas y emitir informes en materia de perfeccionamiento del régimen de inversiones de los Fondos de Pensiones, por propia iniciativa o a petición de la Superintendencia;

4) Pronunciarse sobre temas relacionados con las inversiones de los Fondos, consultadas por los Ministerios de Hacienda y del Trabajo y Previsión Social;

5) Entregar una memoria anual de carácter público al Presidente de la República, dando copia a ambas Cámaras del Congreso, dentro del primer cuatrimestre de cada año, y

6) Encargar estudios técnicos con relación a las inversiones de los Fondos de Pensiones.

El Consejo está integrado por un miembro designado por el Presidente de la República; uno por el Consejo del Banco Central; un miembro designado por las AFP, y dos designados por los Decanos de las Facultades de Economía o de Economía y Administración de las Universidades que se encuentren acreditadas. Los Consejeros duran 4 años en sus cargos y puede renovárseles su designación o ser reelegidos, según del caso, por un nuevo período consecutivo, por una sola vez.

Podemos constatar que este Consejo Técnico posee una importante influencia en la administración de las inversiones que efectúen las AFP, pero en su composición no se incluye a los afiliados. Los representantes de los asegurados tienen una voz en la Comisión de Usuarios con facultades indicativas y propositivas. 
El "Consejo Consultivo Previsional", obra también de la reforma de 2008, otorga asesoría a los Ministros del Trabajo y Previsión Social y de Hacienda en las materias relacionadas con el Sistema de Pensiones Solidarias.

Entre las funciones del Consejo se cuentan: asesorar sobre propuestas de modificaciones legales de los parámetros del sistema solidario y ajustes a los reglamentos; dar opinión en todo lo que incida en el otorgamiento, revisión, suspensión y extinción de beneficios; evacuar un informe anual remitido a los Ministros del Trabajo y Previsión Social y de Hacienda y al Congreso Nacional, que contenga su opinión acerca del funcionamiento de la normativa sobre el sistema de pensiones solidario.

Entre las opiniones que emite el Consejo figura la relativa a los impactos en el mercado laboral y los incentivos al ahorro, y los efectos fiscales producidos por las modificaciones de normativas del sistema solidario.

Adicionalmente, el Consejo da su opinión respecto de todas las materias relativas al sistema solidario en que los Ministros indicados pidan su parecer. Por su parte, los Ministros deberán emitir una respuesta formal a cada Informe elaborado por el Consejo. En determinados casos, como Informes sobre reformas legales y reglamentarias, los Ministros remitirán dicho documento, así como la respuesta recién indicada, al Congreso Nacional.

El Consejo está integrado por un miembro designado por el Presidente de la República, que lo preside, y cuatro designados por el mismo Presidente y ratificados por el Senado, los cuales durarán seis años en sus funciones. Los consejeros deben ser personas con reconocido prestigio por su experiencia y conocimientos en el campo de la Economía, el Derecho y disciplinas relacionadas con la seguridad social y el mercado laboral. Los consejeros designados con ratificación del Senado son inamovibles en sus cargos.

En definitiva, los afiliados, beneficiarios o usuarios se encuentran representados en uno de los tres organismos colegiados existentes en el sistema: la Comisión de Usuarios. Esta Comisión posee un carácter propositivo a partir de las consideraciones que efectúan sus integrantes.

\subsection{QUE LOS EMPLEADORES CONTRIBUYAN AL FINANCIAMIENTO DEL SISTEMA}

En el financiamiento de las prestaciones de vejez, invalidez y sobrevivencia, en el régimen de capitalización individual, originalmente los empleadores no participaban de ninguna forma; era el trabajador quien contribuía en forma exclusiva con el $10 \%$ de su remuneración al financiamiento de su futura pensión, más el pago de una cotización adicional de cuantía variable, ${ }^{8}$ para solventar un seguro de invalidez y sobrevivencia más la comisión a la AFP, pero el empleador no realizaba una cotización 'en conjunto con el trabajador para financiar los fondos que se acumulaban en la cuenta de capitalización individual.

Sin embargo, entre los diversos perfeccionamientos que ha experimentado el régimen por capitalización desde su establecimiento en 1980 , se encuentra el relativo a la

\footnotetext{
${ }^{8}$ A enero de 2010 , la Comisión por depósitos en promedio es de $1,5 \%$, en tanto que la cotización o aporte para el seguro de invalidez y sobrevivencia es de $1,87 \%$. A contar de mediados de 2010 y a propósito de la licitación de afiliados, establecida por la Ley $\mathrm{N}^{\circ} 20.255$, los nuevos afiliados por 24 próximos meses deberán incorporarse en al AFP licitada, en éste caso la Administradora MODELO, que tendrá una comisión de $1,14 \%$ por depósitos (artículos: 160 y ss. del DL $N^{\circ} 3.500$ ).
} 
situación de pensión de las personas que realizan trabajos que califican como pesados y que, por consiguiente, sufren un agotamiento anticipado o más acelerado de las fuerza físicas o intelectuales, que hace recomendable adelantar la edad de retiro, en relación con la regla general. En 1995 se estableció que parte de la mayor cotización que se empezó a exigir a contar de esa fecha para financiar pensiones de ese tipo, es de responsabilidad del empleador, lo que significó alterar una de las bases iniciales del régimen en este aspecto9. La cotización extrafinanciada por el empleador para el caso de los trabajos pesados está sujeta a una reglamentación estricta y solo beneficia al grupo de afiliados cuyos puestos de trabajo califican como de este tipo.

Enseguida, con la reforma de 2008, el empleador ha empezado a responder de la cotización al seguro de invalidez y sobrevivencia de sus trabajadores. La parte de la cotización adicional destinada al financiamiento de este seguro se entera por el empleador, de manera proporcional al monto que estos paguen por concepro de remuneraciones imponibles al respectivo trabajador, sobre el total de dichas remuneraciones. Se exceptúan los trabajadores jóvenes, que reciban una bonificación previsional ${ }^{10}$. Esta reforma está en general en aplicación a contar del 1 de julio de 2009. Excepcionalmente y hasta junio de 2011, se encuentran exentos de cumplirla los empleadores que durante el respectivo mes declaren cotizaciones previsionales por menos de 100 trabajadores, período durante el cual la cotización adicional del correspondiente afiliado deberá incluir el pago de la cotización destinada al financiamiento del seguro. Esta excepción no se aplica a los "Órganos del Estado"11. En definitiva, al cabo del proceso de aplicación gradual de la reforma, el empleador se hará cargo del total de la cotización adicional, que permite financiar el seguro de invalidez y sobrevivencia de los afiliados, y por consiguiente, contribuir decisivamente en la financiación de eventuales pensiones invalidez y sobrevivencia del régimen de capitalización individual.

Adicionalmente, la reforma de 2008 introdujo una figura antes inexistente en la legislación de seguridad social chilena, consistente en el ahorro previsional colectivo. Este mecanismo se asemeja a los planes de fondos de pensiones de tipo 401(k) y contienen la posibilidad de que el empleador realice una contribución equivalente o superior a la que haga el trabajador ${ }^{12}$ (match contribution). Este mecanismo de ahorro exige un gran diálogo y participación entre trabajadores y empleador, para poder obtener beneficios suficientes. Además, para que produzca un efecto importante en la población activa debe existir un nivel de negociación colectiva alto, situación que no ocurre en Chile ${ }^{13}$. Debemos señalar que la introducción de este mecanismo constituye un avance en cuanto a la aportación del empleador. Cabe agregar que el DL 3.500 también contempla la figura de los Depósitos Convenidos ${ }^{14}$, en virtud de los cuales el afiliado puede depositar en su cuenta individual

\footnotetext{
${ }^{9}$ La Ley $\mathrm{N}^{\circ} 19.404$ introduce modificaciones al DL $\mathrm{N}^{\circ} 3.500$, y dicta normas relativas a pensiones de vejez, considerando el desempeño de trabajos pesados, 1995.

10 Artículol 6 en relación con el artículo 59, ambos del DL N³.500 de 1980. Modificación contenida en el No 8 b) del artículo 91, de la Ley $\mathrm{N}^{\circ} 20.255$

11 Artículos 47 y 48 transitorios de la Ley $N^{\circ} 20.255$.

12 Incluso el trabajador puede no efectuar ninguna, según se convenga.

13 Ver ENCLA 2008 (Dirección del Trabajo), publicada en enero 2009.

14 Artículo 20, inciso tercero, DL N³.500.
} 
las sumas que haya convenido con su empleador con el objeto de reunir el saldo suficiente para financiar una pensión anticipada o de incrementar el monto de su pensión.

De esta forma, existe cada vez una mayor participación del empleador en el financiamiento del régimen, solo está excluido legalmente de contribuir al financiamiento de la cuenta individual, pero por la vía de ahorro previsional colectivo puede incidir con su aporte de forma relevante al financiamiento de la pensión.

A modo de conclusión, la reforma de 2008, incluso antecedentes legislativos anteriores a ella, han introducido importantes modificaciones a la cobertura y a la estructura del sistema chileno de protección de la vejez, invalidez y sobrevivencia, que permitirán una mirada diferente a las tres observaciones de la Comisión de Expertos de la OIT. A partir de esta reforma el régimen de capitalización individual deja de ser la única referencia de cobertura y debe observarse la protección que se otorga a través del régimen solidario de pensión de financiamiento estatal. Además, la seguridad social se encuentra en permanente cambio (es una de sus características) y sus instituciones se adaptan con frecuencia a las nuevas realidades. Esos cambios en el futuro permitirán revisar con otros antecedentes las observaciones que nos preocupan.

En definitiva, los cambios experimentados por el sistema chileno de pensiones en los últimos años, en particular la reforma de 2008, estimamos que impactarán en la evaluación de las alegaciones de la OIT, de una forma que seguramente pueden llevar a su revisión.

Asimismo, no se puede dejar de mencionar que la última reforma incluye aspectos que hacen al sistema chileno más equilibrado en la relación pública privada.

Santiago y París, febrero de 2010. 